\title{
Estimação espectral do sinal de vibração para o monitoramento do desgaste do dressador de ponta única
}

\author{
Spectral estimation of vibration signal \\ for monitoring the wear of \\ single-point dresser
}

\author{
Pedro de Oliveira Conceição Junior ${ }^{1}$, Marcelo Marchi ${ }^{2}$, Cesar Henrique Rossinoli Martins ${ }^{1}$, \\ Doriana D’addona $^{3}$, Paulo Roberto de Aguiar ${ }^{1}$, Eduardo Carlos Bianchi ${ }^{2}$,
}

\footnotetext{
${ }^{1}$ Universidade Estadual Paulista - UNESP - Campus de Bauru. Departamento de Engenharia Elétrica, Av. Luiz Edmundo Carrijo Coube, 14-01, Caixa Postal 473, CEP 17033-360, Bauru, SP, Brasil.

${ }^{2}$ Universidade Estadual Paulista - UNESP - Campus de Bauru. Departamento de Engenharia Elétrica, Av. Luiz Edmundo Carrijo Coube, 14-01, Caixa Postal 473, CEP 17033-360, Bauru, SP, Brasil.

${ }^{3}$ Università Degli Studi di Napoli Federico II - Napoli - Dipartimento di Chimica, Materiali e Ingegneria della Produzione Industriale COINOR - Corso Umberto I, 40, 80138 Napoli, Itália.

e-mail:pedroliveira@hotmail.com; marcelo.marchi@outlook.com; cesar.martins@outlook.com; daddona@unina.it; aguiarpr@feb.unesp.br; bianchi@feb.unesp.br
}

\section{RESUMO}

O uso de dressadores desgastados pode proporcionar uma menor agressividade para o rebolo, causando um aumento das forças de corte e a perda mais rápida de afiação dos grãos que por consequência compromete a qualidade final da peça a ser usinada. Por outro lado, um método de monitoramento do processo de dressagem em tempo real, que permita identificar o momento em que o dressador esteja desgastado, e assim seja substituído, é algo que pode ser de grande importância para o processo de retificação, visando garantir a qualidade e precisão das peças usinadas. Dessa forma, esse trabalho teve como objetivo avaliar o desgaste do dressador de ponta única, por meio da análise espectral do sinal de vibração durante o processo de dressagem. Para isso, ensaios de dressagem foram realizados, utilizando uma retificadora plana e variáveis de entrada um rebolo de óxido de alumínio, um dressador de ponta única de diamante sintético do tipo CVD (Chemical Vapour Deposition), profundidade de dressagem de $40 \mu \mathrm{m}$; velocidade do dressador de 3,45 mm/s e o grau de recobrimento $U_{d}$ igual a 1 no início da dressagem. As variáveis de saída foram a aquisição dos sinais de vibração por meio de um acelerômetro fixo no suporte do dressador, coletados por um osciloscópio e as medições do desgaste do dressador por meio de um microscópio. Por meio da análise espectral do sinal de vibração, em três condições de uso, sendo, nova, meia vida e desgastado, utilizando o método Welch e a Transformada Rápida de Fourier (FFT), foi possível identificar faixas de frequência entre $1 \mathrm{kHz}$ e $8 \mathrm{kHz}$, em que a amplitude aumentava em função do desgaste do dressador. Foi constatado que o aumento da atividade de vibração é diretamente proporcional ao desgaste do dressador CVD. Foi possível identificar faixas de frequências que melhor caracterizam tal situação, como nos trechos de $2 \mathrm{kHz}$ a $4 \mathrm{kHz}$ e de $6 \mathrm{kHz}$ e $8 \mathrm{kHz}$. Os resultados indicam a possibilidade de implementação de um sistema de monitoramento em tempo real, a partir do uso de filtros digitais nessas faixas de frequência.

Palavras-chave: Operação de dressagem; dressador CVD; desgaste do dressador; vibração; análise espectral; monitoramento.

\section{ABSTRACT}

The use of dressers worn can provide a less aggressive to the grinding wheels, causing an increase in cutting forces and a more rapid loss of grinding grain consequently compromise the final quality of the workpiece to 
be machined. On the other hand, a method of monitoring the dressing operation in real time, identifying the moment in which the dresser is worn and thus to be replaced, something which can be of great importance for the grinding process in order to assure quality and accuracy of the workpieces. Thus, this study aimed to evaluate the wear of the single point dresser, through spectral analysis of vibration signal during the dressing operation. For this dressing assays were performed using a flat grinding and input variables a grinding wheel aluminum oxide, one the single point dresser of synthetic diamond $r$ type CVD (Chemical Vapour Deposition), depth of dressing $40 \mu \mathrm{m}$, dresser speed of $3.45 \mathrm{~mm} / \mathrm{s}$ and the degree of coating $U_{d}$ equal to 1 at the beginning of dressing. The output variables were the acquisition of the vibration signals via an accelerometer fixed in the dresser holder, collected by an oscilloscope and dresser wear measurements using a microscope. Through spectral analysis of vibration signal in three conditions of use, and, new, half-life and worn, using the Welch method and Fast Fourier Transform (FFT), it was possible to identify the frequency bands between $1 \mathrm{kHz}$ and $8 \mathrm{kHz}$ in the amplitude increased according to the dresser wear. It has been found that the increased vibration activity is directly proportional to the CVD dresser wear. It was possible to identify frequency bands that best characterize such a situation, as in bands of $2 \mathrm{kHz}$ to $4 \mathrm{kHz}$ and $6 \mathrm{kHz}$ and $8 \mathrm{kHz}$. The results indicate the possibility of implementing a real-time monitoring system, from the use of digital filters in these frequency bands.

Keywords: Dressing operation; dresser CVD; dresser wear; vibration; spectral analysis; monitoring.

\section{INTRODUÇÃO}

"Retificação é dressagem" é o slogan mantido na comunidade do processo de retificação. Indica, além de todos os outros parâmetros do processo, a importância da tecnologia de condicionamento de rebolos, nos resultados de fabricação. O processo de condicionamento do rebolo, que consiste em dressagem e limpeza, determina a taxa de remoção de material, as forças na retificação, a qualidade da superfície e as propriedades dos materiais na zona de subsuperfície [1]. A retificação é um processo de fabricação complexo influenciado por muitos fatores, como a peça, a máquina, o rebolo e a configuração do processo. O rebolo, o qual é a ferramenta de corte, é o único fator que diferencia o processo de retificação dos outros processos de usinagem. É conhecido o fato de que a topografia e as condições nas quais o rebolo é preparado exercem profunda influência sobre o desempenho da retificação, o que é evidenciado pelas forças de corte, energia consumida, temperatura na zona de corte e muitas vezes no acabamento da peça [2]. E, além disso, a superfície do rebolo desempenha um importante papel na rugosidade das peças [3].

Segundo Marinescu et al. [4], dressagem é o processo de condicionamento da superfície do rebolo visando sua remodelação quando o mesmo perdeu sua forma original pelo desgaste. É a operação conjunta de perfilamento e afiação dos rebolos convencionais, tendo como objetivo restaurar a eficiência de corte da ferramenta. Para manter as características e funcionalidades do rebolo, é necessário realizar periodicamente a dressagem, garantindo uma topografia adequada da sua superfície de corte Essa topografia influencia na rugosidade e na camada superficial da peça por meio da quantidade e forma dos seus grãos abrasivos, do volume dos poros e do comportamento do desgaste da camada abrasiva [5] e, por conseguinte, na vida do rebolo, no tempo de ciclo de retificação e a qualidade final da peça [4]. O processo de dressagem é um elemento transformador da topografia do rebolo, que deve ser muito bem conhecido para que se tenha um maior controle sobre do seu desempenho. $\mathrm{O}$ uso de dressadores desgastados pode proporcionar uma menor agressividade para o rebolo, causando um aumento das forças de corte e a perda mais rápida de afiação dos grãos. $\mathrm{O}$ controle da operação do rebolo e a eliminação de condições indesejáveis podem ser detectados por meio de um sistema online de monitoramento. Por isso, o desenvolvimento de um sistema de monitoramento e controle do processo, em tempo real, é de fundamental importância [6].

Durante pesquisas sobre dressagem realizadas por grupos anteriores a este trabalho, se constatou uma ausência de métodos indiretos de monitoramento do desgaste de dressadores de ponta única. Além dessa questão existe também a preocupação com a seleção correta do material e a sua relação custo e beneficio. O diamante natural tem sido muito aplicado em dressadores de ponta única, devido às características físicoquímicas desse material. Contudo, a desvantagem do diamante natural para o uso industrial é o seu elevado custo [7] [8]. O grande interesse pela síntese de diamante é de replicar as propriedades físico-químicas do diamante natural, de modo que o diamante sintético apresente elevada dureza, baixa densidade, baixo coeficiente de expansão térmica, alto índice de refração, alta condutividade térmica à temperatura ambiente, excelente isolante elétrica, inércia química [9]. Porém, as propriedades mecânicas, elétricas e óticas das películas dos diamantes sintéticos se aproximam daquelas do diamante natural, mas ainda não de forma satisfatória [7], reforçando assim, a necessidade do aprimoramento do processo, realizando o monitoramento e automação do mesmo. 
Um dos métodos empregados no monitoramento do processo de retificação e, particularmente, no monitoramento da dressagem, é o sistema de monitoramento por sensores, que deve ser capaz de identificar problemas no processo com alta confiabilidade. Alguns sensores usados atualmente para fazer tal monitoramento, como o sensor de vibração, por exemplo, estão cada vez mais presentes nos trabalhos que buscam melhorar sua qualidade e produtividade. Quando manuseados de maneira correta, os dados coletados pelos sensores fornecem informações importantes para a deteç̧ão de problemas em máquinas e processos, assim como para a análise de produtividade ou estado de produção [10].

No processo de retificação e, particularmente, na operação de dressagem, ocorrem tanto vibrações forçadas como as autoexcitadas. Vibrações forçadas são causadas pela rotação de eixos desbalanceados, colisões entre elementos no final dos seus movimentos, etc. Vibrações autoexcitadas são causadas por colisões entre grãos abrasivos e partículas duras da peça, espessura de cavaco variável, atrito e mecanismo da formação de cavaco [11]. Acelerômetros são usados no diagnóstico de máquina e também em monitoramento de processos de usinagem. O processamento digital do sinal de vibração tem como objetivos eliminar ruídos e extrair características desejáveis do sinal [12]. O sinal de vibração também pode ser estudado pela análise no domínio da frequência. O espectro de frequência fornece, em muitos casos, a informação detalhada sobre a condição de sinais que não pode ser obtida no domínio tempo. O conteúdo de frequências pode ser obtido pela aplicação de métodos e ferramentas como as transformadas de Fourier [13].

Diversos estudos sobre monitoração da operação de dressagem têm sido publicados, poucos destes, porém, são focados no desgaste de dressadores de ponta única. D’Addona et al., [14] propuseram um método para caracterizar o estado de desgaste de ponta única de diamante sintético utilizando sinais de vibração através da aplicação de Redes Neurais Artificiais ( RNAs). Os resultados mostraram um método eficaz para o monitoramento do desgaste em dressadores com base em análise de sinais de vibração. A pesquisa de Miranda et al., [15] apresentou um método para prever o nível de desgaste do dressador de ponta única com base em sinais de emissão acústica (EA) e vibração utilizados como variáveis de entrada para modelos fuzzy. Os resultados indicam que os modelos fuzzy utilizando as estatísticas derivadas dos sinais são altamente eficazes para prever o nível de desgaste da dressador de ponta única, porém limitados para rebolos de óxido de alumínio. O estudo de Zhang \& Shin [16], focou as propriedades de dressadores de ponta única na dressagem de rebolos CBN utilizando laser. Seus resultados indicam que o desgaste dos dressadores depende da potência do laser, assim como da profundidade e do avanço do dressador. Os autores também concluíram que o aquecimento influencia a taxa de desgaste do dressador. Habrat et al., [10] descreve um sistema de monitoramento para medir o raio equivalente do dressador de diamante, calculando assim seu desgaste.

Moia et al., [17] monitoraram a operação de dressagem com estatísticas derivadas do sinal de EA que serviram de entrada para o modelo de RNA. Os resultados foram bem sucedidos para classificar a condição do rebolo após a operação de dressagem, como sendo o mesmo afiado (com capacidade de corte) e em função do uso, como empastado (com perda da capacidade de corte). Martins et al., [18] [19] descreveram um estudo para classificar o desgaste de dressadores de ponta única utilizando os valores médios quadráticos (RMS) dos sinais de emissão acústica, e redes neurais artificiais. Seus resultados mostraram uma taxa de classificação satisfatória, porém, a um custo computacional muito alto. Os trabalhos de Souza et al., [20], [21] foram direcionados para a estimação da condição de desgaste de um dressador de ponta única. Arquiteturas de RNAs foram usadas para classificar o desgaste do dressador, cujas entradas foram obtidas com base no processamento digital de EA e vibração. Observou-se no estudo que o conteúdo harmônico dos sinais de emissão acústica e vibração sofrem influência da condição de desgaste e, quando bandas de frequências selecionadas são usadas como entradas das redes neurais bons resultados foram obtidos, com até 98,3\% de taxa de acertos pela rede.

Dada à importância do monitoramento da operação de dressagem e da necessidade crescente de automação do processo de retificação, o presente trabalho estuda o desgaste do dressador de ponta única CVD, por meio da análise espectral do sinal de vibração durante o processo de dressagem, baseando-se no trabalho de Martins et al. [18] [19] e Souza et al., [20], [21]. A diferença é que o presente trabalho estuda o desgaste dos dressadores por meio da estimação espectral ao invés de redes neurais artificiais. Para tanto, foi utilizado o método Welch partindo da FFT para posterior comparação. A justificativa de se utilizar esse método parte da hipótese de que, sistemas inteligentes como RNAs apresentam custo computacional elevado e métodos ópticos além do elevado custo são demorados. E empregando a estimação espectral no tratamento de sinais é possível identificar as melhores faixas de frequência, que possivelmente se correlacionam com as condições do processo para posterior implementação de filtros digitais nessas faixas, sendo que essa implementação na prática apresenta baixo custo e ao mesmo tempo apresenta eficiência. Com o presente estudo, um sistema de monitoramento é proposto para permitir a melhor seleção dos dressadores em função de seu desgaste, contribuindo para a automatização do processo. 


\section{OPERAÇÃO DE DRESSAGEM}

Dressagem é o processo de condicionamento do rebolo, que permite restabelecer as características e formato originais perdidos devido ao desgaste em função do uso, a fim de manter a precisão, exatidão e a qualidade do processo de retificação[22]. Entender a operação de dressagem é um fator crítico para a obtenção de ótimo processo de retificação [4]. Inicialmente a topografia e forma macroscópica do rebolo são geradas pela preparação do mesmo por meio da dressagem [5]. Segundo Lin, Liu, \& Huang [23] a topografia do rebolo é caracterizada pelo número de arestas de corte. De modo que, essa topografia tem influência sobre a geometria do cavaco produzido e no processo de retificação como um todo. Pode-se então considerar que o processo de retificação engloba tanto a dressagem como a própria retificação.

Do ponto de vista de remoção do material, as propriedades estáticas mais importantes da topografia do rebolo são os parâmetros relacionados à aresta de corte e sua distribuição. O dressador de ponta única carrega um único diamante, que deve formar um ângulo de ataque limpo em relação ao rebolo para que o processo de dressagem seja eficiente. Se o diamante estiver perpendicular ao rebolo durante a dressagem, ele será gasto rapidamente. Se o ângulo do diamante for igual ao da superfície do rebolo a região de contato irá gerar muito calor, podendo danificar o diamante [19]. O dressador de ponta única carrega um único diamante, que deve formar um ângulo de ataque limpo em relação ao rebolo para que o processo de dressagem seja eficiente. Se o diamante estiver perpendicular ao rebolo durante a dressagem, ele será gasto rapidamente. Se o ângulo do diamante for igual ao da superfície do rebolo a região de contato irá gerar muito calor, podendo danificar o diamante [24]. O dressador de ponta única é usualmente instalado com um ângulo relativo entre a ferramenta e o rebolo de $\alpha_{d}$ entre $10^{\circ}$ e $15^{\circ}$ [5]. O ângulo é mostrado na Figura 1.

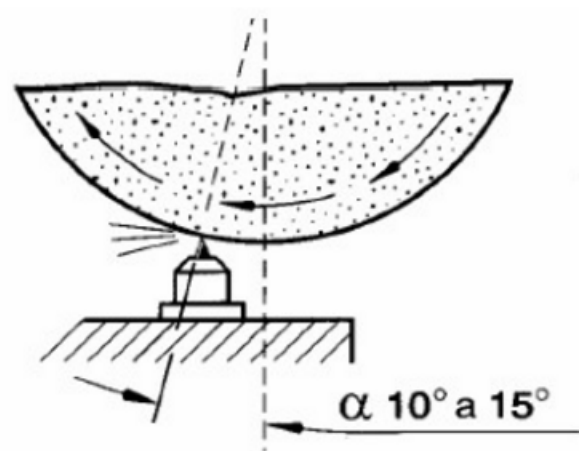

Figura 1: Ângulo relativo entre a ferramenta e o rebolo, [25].

Dentre os materiais o diamante é aquele que apresenta a maior condutividade térmica a temperatura ambiente, sendo quatro vezes superior a do cobre ou da prata, é um bom isolante elétrico, possui uma constante dielétrica pequena, um coeficiente de desgaste e de expansão térmica pequeno, elevada resistência química e de corrosão para a maioria dos ácidos e substâncias oxidantes, e é opticamente transparente [26]. A grande desvantagem do diamante natural para o uso industrial é o seu elevado custo [7]. O grande interesse pela síntese de diamante é de replicar as propriedades físico-químicas do diamante natural, de modo que o diamante sintético apresente elevada dureza, baixa densidade, baixo coeficiente de expansão térmica, alto índice de refração, alta condutividade térmica à temperatura ambiente, excelente isolante elétrica, inerte quimicamente, apresenta compatibilidade biológica e quando dopado torna-se um bom semicondutor [9].

A partir da década de 70 começaram a surgir trabalhos acadêmicos que buscavam obter pequenas camadas de diamante através de gases compostos por carbono. Essas camadas, denominadas filmes, eram produzidas em pressões inferiores a uma atmosfera e em temperaturas abaixo de $1200 \mathrm{~K}$, em substratos incrustados com pó de diamante. A partir disso, a técnica de obtenção de diamante a partir de um gás precursor se disseminou pelo mundo, como um método mais fácil e produtivo do que aquele utilizado nas décadas de 50 e 60. Os diamantes sintéticos criados por está técnica passaram a se chamar de diamante CVD (Chemical Vapour Deposition) [9]. Contudo, as propriedades mecânicas, elétricas e óticas das películas dos diamantes CVD se aproximam daquelas do diamante natural, mas ainda não de forma satisfatória [7].

\section{MONITORAMENTO DO PROCESSO DE RETIFICAÇÃO}

O desenvolvimento de um sistema de monitoração e controle em tempo real é de fundamental importância para automação dos processos de usinagem, a fim de que se tenha um processo capaz de estabelecer o momento da troca de uma ferramenta, realizar a sua troca e realizar mudanças nos parâmetros de operação visando a otimização do processo [27]. Segundo Xue \& Naghdy [28] a ponta do dressador é um fator que afeta significativamente a rugosidade da peça quando comparado com outros parâmetros do processo de retifica- 
ção, tais como profundidade de corte e velocidade da mesa. O monitoramento dos parâmetros de dressagem resulta em valores mais consistentes de rugosidade para a operação de retificação. Erros de dressagem podem afetar diferentemente a qualidade da dressagem devido a flutuações da profundidade real de dressagem, desgaste do dressador e outros distúrbios. Um sistema de monitoramento pode detectar essas condições indesejáveis.

Alguns sensores usados atualmente para fazer tal monitoramento, como o sensor de vibração, por exemplo, estão cada vez mais presentes nos que buscam melhorar sua qualidade e produtividade. Quando manuseados de maneira correta, os dados coletados pelos sensores fornecem informações importantes para a deteç̧ão de problemas em máquinas e processos, assim como para a análise de produtividade ou estado de produção [10]. Os dados coletados pelos sensores são submetidos à análise por meio do processamento digital de sinais, que consiste em se aplicar transformações a um sinal analógico, ou digital, com o objetivo de eliminar ruídos e extrair características. Algumas operações comumente utilizadas no processamento de sinais são: ampliação, filtragem, transformações matemáticas, integração de sinais, conversão analógico para digital.

Quando se deseja obter uma melhor compreensão das ocorrências nos dados, utiliza-se o espectro de frequência, que fornece em muitos casos, a informação detalhada sobre a condição de sinais que não pode ser obtida no domínio tempo. O conteúdo de frequências pode ser obtido pela aplicação de métodos e ferramentas como as transformadas de Fourier [13]. Uma análise do espectro de vibração pode ser baseada na seleção de faixas de frequência que caracterizam a variável física que se deseja controlar ou estimar no processo. Um dos métodos utilizados na análise em frequência do sinal é a Transformada Rápida de Fourier (FFT - Fast Fourier Transform). A FFT é um método de computar a Transformada Discreta de Fourier (DFT - Discrete Fourier Transform) de maneira eficiente, com menor custo computacional e o menor tempo de processamento [29].

Por meio da Transformada de Fourier é possível obter a Densidade Espectral de Potência (PSD - Power Spectral density) de um sinal, que mostra justamente como a potência do mesmo está distribuída ao longo de todas as suas componentes de frequência. A estimação espectral clássica consiste nos métodos de obtenção da estimativa da PSD sem parametrizar o espectro do sinal. O estimador da PSD por um método direto é conhecido como periodograma, tomando-se o valor esperado das Transformadas de Fourier do sinal. Contudo, os métodos de estimação da PSD clássicos, apresentam em alguns casos inconsistência na relação entre a quantidade de amostras e o resultado estimado. O método Welch tem surgido para resolver esse problema, sendo um método de periodogramas usado para determinar a densidade de potência dos componentes de frequência de um sinal, baseado na FFT que permite a sobreposição dos segmentos de dados e passando cada segmento por uma janela. Welch propôs o uso da janela de Hanning com $50 \%$ de sobreposição e a sua variância do periodograma é inversamente proporcional ao número de segmentos no qual o sinal é dividido [30].

Para obter o PSD de um sinal pelo Welch, divide-se em quadros como 64, 128 e 256, que são potências de dois. Para avaliar o espectro de potência, este método separa os dados em vários segmentos sobrepostos, calcula um espectro de potência usando uma FFT sobre cada segmento e, em seguida, as médias destes espectros [30]. O periodograma de Welch é dado pela média dos periodogramas originais de todos os $P$ segmentos do sinal ponderados por uma janela. Mais informações sobre o método são encontradas em [31]-[33].

No processo de retificação ocorre tanto a vibração autoexcitada, ou trepidação, quanto a vibração forçada. Vibrações forçadas são geradas por distúrbios periódicos externos ao processo. Podem ter origem em eixos ou rebolos desbalanceados, motores elétricos, rolamentos, atuadores hidráulicos, ou até mesmo por outras máquinas nas adjacências [34]. A principal dificuldade deste tipo de vibração é sua eliminação, já que a identificação da fonte de vibração é relativamente simples, por meio de medições das frequências [35]. Já as causas da vibração autoexcitada são muito mais complexas, pois estão associadas à vibração natural da estrutura máquina-ferramenta. A instabilidade da retificação é atribuída a aspectos relacionados ao rebolo e à peça [36].

Drew et al. [37], afirmam que a frequência de trepidação está relacionada com a frequência natural fundamental transversal da peça e do rebolo. Deste modo, se a largura de retificação não for muito maior que a largura limite para uma retificação estável, a frequência de trepidação é a próxima harmônica inteira da frequência do rebolo acima da frequência natural transversal da peça. Inicialmente, a topografia do rebolo é gerada pela operação de dressagem e as mudanças ocorrem ao longo da retificação. O desgaste de rebolos pode ser muito influenciado pelos níveis de vibração existentes no processo. Dessa forma os níveis de vibração também podem influenciar diretamente a operação de dressagem, bem como o desgaste do dressador. Apesar de esta influência ser importante, ainda há poucos estudos sobre esse assunto na literatura. Sendo assim, o seu estudo no âmbito deste trabalho será enfatizado.

O monitoramento da vibração do processo de retificação é usado principalmente para medir vibrações 
forçadas para identificar desbalanceamento ou fontes externas de vibração; e, encontrar uma correlação com as condições do processo por meio do monitoramento indireto por acelerômetro, estudando, dessa forma, o sinal de vibração [38]. A vibração forçada é facilmente detectada, pois sua frequência pode ser mensurada e comparada com os elementos rotativos da própria máquina ou de equipamentos adjacentes a esta. Já a vibração autoexcitada possui frequências mais altas, próximas da frequência de ressonância da máquina e sua importância no processo de retificação é maior uma vez que a sua origem é mais difícil de ser determinada. Diversos trabalhos foram realizados com o intuito de utilizar os sinais provenientes da retificação para o monitoramento e/ou diagnóstico do processo. Hassui et al. [11] utilizou o sinal de vibração para a avaliação do processo de retificação. O sinal de vibração do processo foi utilizado com sucesso na determinação indireta do melhor momento para a dressagem do rebolo. Foi verificado ainda que a vibração seja capaz de indicar com clareza as três fases da retificação cilíndrica externa de mergulho: deformação elástica inicial, remoção de material e spark-out.

Em um estudo David \& Warneck [39] apud Hassui et al. [11] relacionaram o sinal de vibração com as forças dinâmicas da retificação. A pesquisa mostrou que estas forças fazem o sistema (a máquina o rebolo e a peça) vibrar e que é possível captar o aumento de amplitude da vibração em algumas frequências à medida que o rebolo perde a afiação. Os autores concluíram que vibrações irregulares de pequenas amplitudes são típicas do processo sem a ocorrência de trepidação, já quando ocorrem, as vibrações autoexcitadas são praticamente harmônicas e de altas amplitudes. Na pesquisa de Chuangwen et al., [40] foi usado um método de avaliação da condição de desgaste de uma ferramenta através de redes neurais e análise de pacotes wavelet por meio do sinal de vibração. O método apresentou uma análise de bandas de frequências para o monitoramento do desgaste da ferramenta, podendo ser usado para estimar sua condição durante o uso com boa precisão.

A utilização de um método de análise e estimação como esse em um sistema de monitoramento reflete a otimização do processo e, por isso, a importância da realização desse trabalho em monitorar a operação de dressagem, utilizando a vibração (aceleração) com base no estudo espectral desse sinal. Isso permitirá uma melhor seleção dos dressadores em função de seu desgaste, mantendo assim, as condições ideais de processo

\section{MATERIAL E MÉTODOS}

Um banco de ensaios foi desenvolvido de modo a permitir a análise das condições de desgaste da ferramenta de dressagem a partir dos sinais puros ( $r a w$ ) de aceleração, coletados em um osciloscópio da Yokogawa, modelo DL850, a uma taxa de 2 milhões de amostras por segundo. Os ensaios foram realizados em uma máquina retificadora plana tangencial da Sulmecânica modelo RAPH 1055, para a operação de dressagem de um rebolo convencional de óxido de alumínio tipo 38220KVS e dimensões 355,6 x 12,7 x 127 mm, da fabricante NORTON. Os parâmetros de operação foram adequadamente controlados para assegurar as mesmas condições para todos os dressadores.

Um dressador de ponta única de diamante sintético CVD foi utilizado nos ensaios de dressagem para o estudo do seu desgaste. As medições da ponta de diamante foram realizadas em intervalos de 20 passadas para cada ensaio, utilizando-se de um sistema ótico e software CAD para cálculo da área desgastada. Para obtenção dos sinais de aceleração foi utilizado um sensor fixo, posicionados no suporte do dressador, modelo 353B03 e um módulo condicionador de sinal modelo 482B11, ambos do fabricante PCB Piezotronic. A Figura 2 mostra o banco de ensaios realizados.

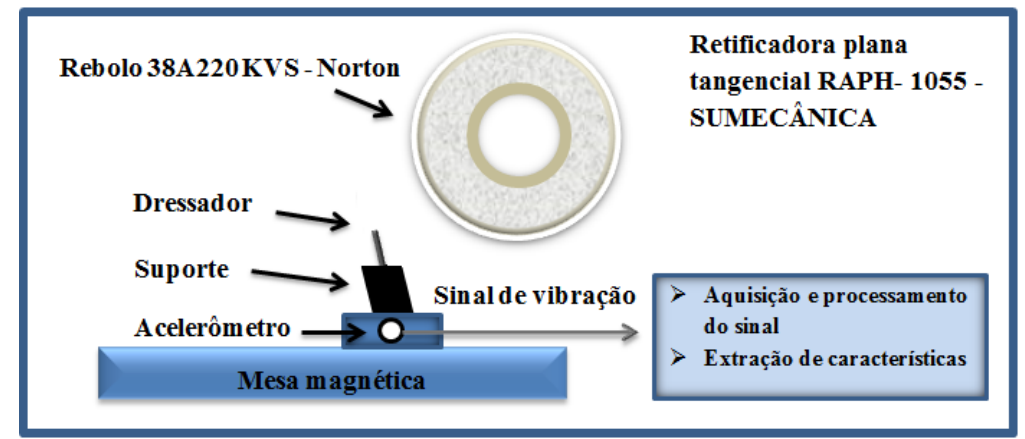

Figura 2: Representação esquemática do banco de ensaios

Os ensaios de dressagem foram realizados com base no trabalho de Martins et al. [18] [19] e Souza et al., [20], [21], consistindo de um número de passadas do diamante pela superfície do rebolo até o fim da vida do dressador, ou seja, até que se observava contato de material metálico com o material do rebolo. Um número de 3 repetições foi adotado para os ensaios de dressagem. A velocidade do dressador foi mantida constante 
em 3,45 mm/s, com profundidade de dressagem constante de $40 \mu \mathrm{m}$, com grau de recobrimento $U_{d}$ igual a 1 no início da dressagem e sem a utilização de fluido de corte para proporcionar um desgaste mais rápido. Com o objetivo de determinar uma correlação entre os sinais de aceleração e a condição de desgaste do dressador, considerou-se inicialmente o conteúdo harmônico dos sinais de aceleração, em diferentes estágios de desgaste.

Para realizar as medições do desgaste da ponta dos dressadores foi utilizado um microscópio estereoscópio modelo CITOVAL 2 com ampliações de 10x a 100x da marca Carl Zeiss Predstavnistvo Beograd (Montenegro), a ampliação adotada para a tomada das imagens foi de 16x. Acoplada a este microscópio estava uma câmera EC 3 da LEICA. A Figura 3 mostra a vista lateral e a frontal da ponta do dressador nos diferentes níveis de desgaste classificados, medidos a cada 20 passadas.

(a)

(b)
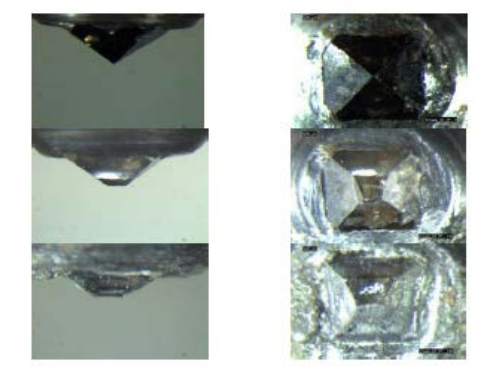

Figura 3: Vista lateral (à esquerda) e frontal (à direita) do dressador CVD, novo (a); meia vida (b) e desgastado (c).

Inicialmente foi encontrada no sinal de vibração a frequência natural da máquina retificadora plana. O estudo é mostrado na Figura 4. Foi estudado o sinal correspondente à primeira passada de dressagem, que naturalmente possui uma condição mais branda, o que permite detectar com mais facilidade, frequências mais baixas no sinal. A Figura 4 (a) mostra o sinal puro de vibração, correspondente a uma passada de dressagem. Na Figura 4 (b) foi calculado a FFT do sinal puro, ainda sem um janelamento definido, pois o objetivo é encontrar a frequência de trepidação da máquina e desconsiderá-la, pois não é o propósito deste trabalho estudar essas frequências. Aplicando um zoom entre 0 e $250 \mathrm{~Hz}$ no gráfico da FFT, conforme mostra a Figura 3 (c) foi possível encontrar picos de frequência no sinal, que são múltiplos de aproximadamente $30 \mathrm{~Hz}$ e $60 \mathrm{~Hz}$, que são respectivamente a frequência do rebolo $(30 \mathrm{~Hz})$, já que sua rotação no processo foi de $1800 \mathrm{RPM}$, e da rede elétrica $(60 \mathrm{~Hz})$.

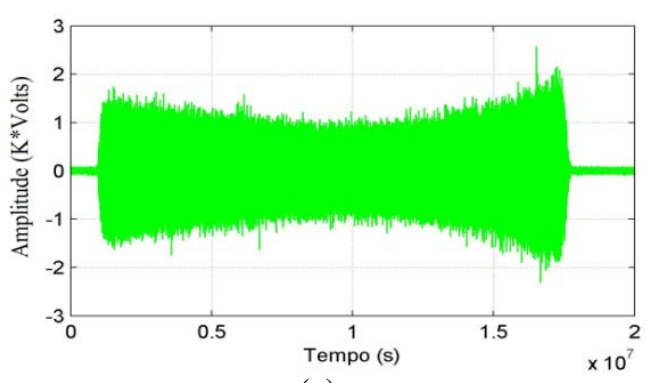

(a)

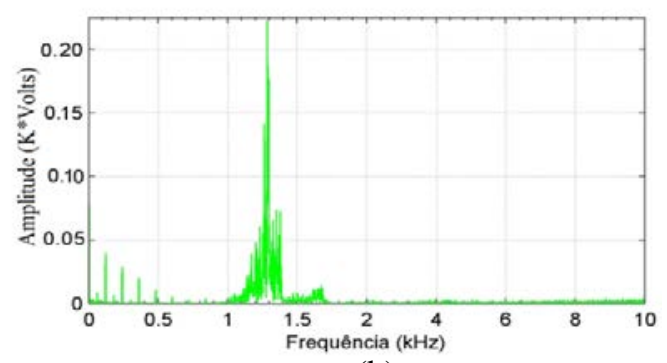

(b)

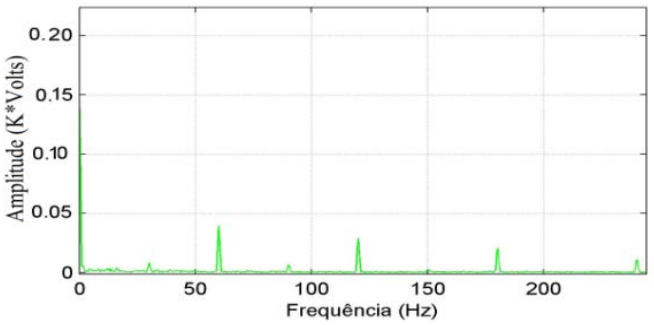

(c)

Figura 4: Sinal puro de vibração da primeira passada de dressagem (a); FFT do sinal (b) e FFT com zoom entre 0 e 250 $\mathrm{Hz}(\mathrm{c})$.

É importante ressaltar que as características dinâmicas, como a frequência natural e frequência de trepidação da máquina foram determinadas com base nas seguintes afirmações: Reddy et al., [48] usou uma análise modal para determinar as características de vibração (frequências naturais e formas de vibração) de 
uma estrutura ou a componente da máquina enquanto ela estiver em operação. De acordo com Reddy et al.,[48] a análise modal para o processo de retificação mostra que a frequência aumenta a cada interação. Esta gama de frequência natural varia de 48,977 Hz a 79,968 Hz. Na pesquisa de Oliveira et al., [49], foram realizados experimentos para observar a influência de vibrações no desgaste do rebolo. Foram aplicadas nessas experiências, deliberadamente, algumas formas de vibração. Estas vibrações senoidais incluídas em frequências naturais, frequência de rotação do rebolo (para simular desequilíbrio) e também ruídos aleatórios. A curva de resposta em frequência foi medida e as frequências naturais de $125 \mathrm{~Hz}, 205 \mathrm{~Hz}$ e $300 \mathrm{~Hz}$ foram encontradas. Desse modo, infere-se que, as frequências naturais encontrados em retificadoras planas encontram-se abaixo de $300 \mathrm{~Hz}$, com base no estudo do comportamento dinâmico.

Através de uma ampliação no gráfico da FFT entre 0 e $310 \mathrm{~Hz}$, conforme mostra a Figura 4, foi possível encontrar picos de frequência no sinal múltiplos de aproximadamente $30 \mathrm{~Hz}$ e $60 \mathrm{~Hz}$, que são respectivamente a frequência do rebolo $(30 \mathrm{~Hz})$, já que sua rotação no processo foi de 1800 RPM, e da rede elétrica (60 $\mathrm{Hz}$ ). De acordo com Reddy [48] e Oliveira et al., [49] em estudos já realizados em busca da frequência de trepidação da máquina e do processo, foi constatado que a mesma deve ser aproximadamente igual à sexta harmônica do rebolo, $(6$ x $30 \mathrm{~Hz})$, ou seja, essa frequência deve ser, para este caso, aproximadamente $180 \mathrm{~Hz}$.

Na sequência foi aplicado nos sinais puros de vibração um filtro passa faixa do tipo Butterworth, de quinta ordem. Para que todas as frequências de trepidação da máquina fossem completamente desconsideradas no estudo, a frequência inicial da faixa foi de $300 \mathrm{~Hz}$ e a frequência final foi $10 \mathrm{kHz}$, que é respectivamente a frequência de corte estipulada pelo fabricante do acelerômetro utilizado nos ensaios. Para a determinação do espectro de frequência foi empregada inicialmente à transformada rápida de Fourier (FFT) com janela de Hanning, considerando-se para efeito de cálculo primeiramente uma janela de $2^{15}$ amostras e, posteriormente, para uma melhor resolução dos sinais, uma janela de $2^{13}$ amostras do sinal pertencentes à passada de dressagem. Em seguida foi calculado o PSD, pelo método Welch, por meio da função Pwelch do software MATLAB, com o mesmo janelamento da FFT, a fim de se comparar os resultados. A análise dos sinais no domínio do tempo foi feita por meio do valor RMS (Root Mean Square) a partir dos sinais filtrados. O RMS foi obtido usando blocos de 2048 amostras do sinal filtrado, o que equivale a uma constante de tempo de 1 ms (milissegundo).

Assim, obtiveram-se as curvas de amplitude e magnitude em função da frequência dos sinais puros filtrados de aceleração, para três condições de desgaste do dressador. As três condições foram classificadas como dressador novo, meia vida e desgastado, representados por "N", "MV" e "D", respectivamente. É importante salientar que as três condições são classificadas em função da escolha dos sinais em diferentes estágios de desgaste calculados pelo sistema ótico. A próxima etapa é verificar se as ferramentas de análise espectrais são capazes de identificar um padrão de comportamento relacionado a essa classificação com base na intensidade dos sinais e, além disso, faixas de frequências relacionadas ao processo.

\section{RESULTADOS E DISCUSSÕES}

A seguir são apresentados os resultados obtidos no experimento de dressagem realizado. Inicialmente serão apresentados os resultados quanto ao cálculo do desgaste do dressador. Em seguida serão apresentados os resultados do estudo do sinal de vibração primeiramente na análise no domínio do tempo e finalmente a análise espectral. Assim, a Figura 5 apresenta os valores médios das áreas desgastadas e seus desvios padrão para cada 20 passadas.

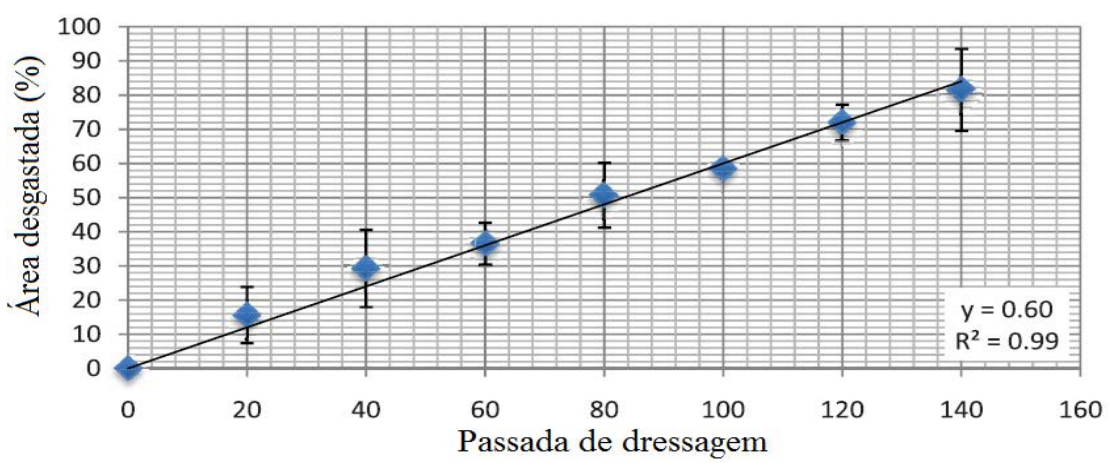

Figura 5: Área desgastada no dressador ao longo das passadas

A partir de observações realizadas nas imagens da vista lateral e frontal do dressador CVD foi possível determinar a sua área desgastada. Devido à presença de impurezas nos diamantes, os ensaios não apresentaram a mesma quantidade de passadas. Dessa forma, para se realizar uma análise do desgaste tendo repeti- 
ção dos dados para o cálculo da média e do desvio padrão para cada medição, foi adotado o total de passadas do menor ensaio como quantidade máxima de passadas. Dessa forma, as passadas até o limite de 140 foram consideradas para análise das áreas para o diamante CVD. Pode ser observada na Figura 5 uma tendência de aumento desgaste ao longo das passadas, conforme o esperado, e um significativo desvio padrão em algumas passadas. Isso pode ser explicada pelas diferenças de friabilidade do dressador (sua capacidade de fraturar o diamante). Embora eles sejam semelhantes, o comportamento do desgaste do diamante varia consideravelmente, devido à vida útil de cada dressador.

Para o processo de classificação do nível de desgaste dos diamantes foram classificadas as passadas até o limite de 20 como dressador novo (N) e como dressador desgastado (D) as passadas em que a área do desgaste lateral ultrapasse aproximadamente em 4 vezes a área observada na passada de número 20. As passadas situadas entre a passada número 20 e a primeira passada da condição desgastada foram classificadas como dressador com meia vida (MV), indicando que nessa região o diamante ainda apresenta condições de operação. Os sinais coletados foram processados a fim de se obter características nas quais o sinal de vibração se correlaciona com o nível de desgaste do dressador. Foi realizada uma análise inicial no domínio do tempo, mostrada na Figura 6, contendo o sinal puro, em seguida o sinal filtrado e por fim o sinal RMS para cada uma das condições do dressador, previamente classificadas.

A partir do sinal puro de vibração foi calculado o RMS, do qual foram extraídos os dados correspondentes ao momento em que o dressador esteve em contato com o rebolo, ou seja, a parte válida da passada, conforme mostrou a Figura 6. A partir desses gráficos é possível observar que dentre os ensaios de um determinado tipo de dressador, existem semelhanças quanto à evolução do nível de vibração conforme o diamante CVD é desgastado. Pode ser notado que o sinal pertencente à condição do dressador desgastada, possui uma atividade vibracional maior, apresentando portando uma amplitude de igual modo. Dessa forma, infere-se que o sinal de vibração contem informações sobre a evolução do desgaste da ferramenta.
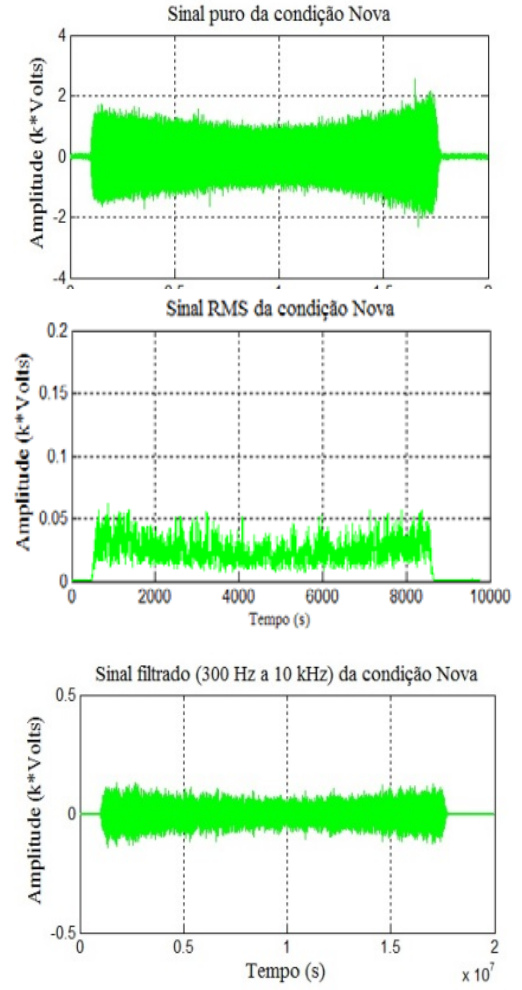

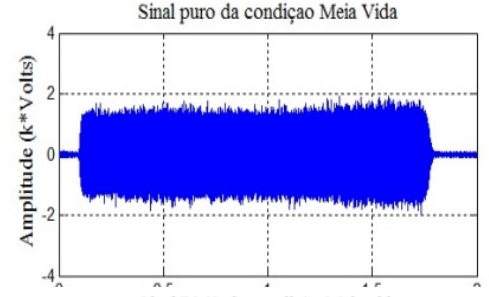

Sinal RMS da condição Meia vida
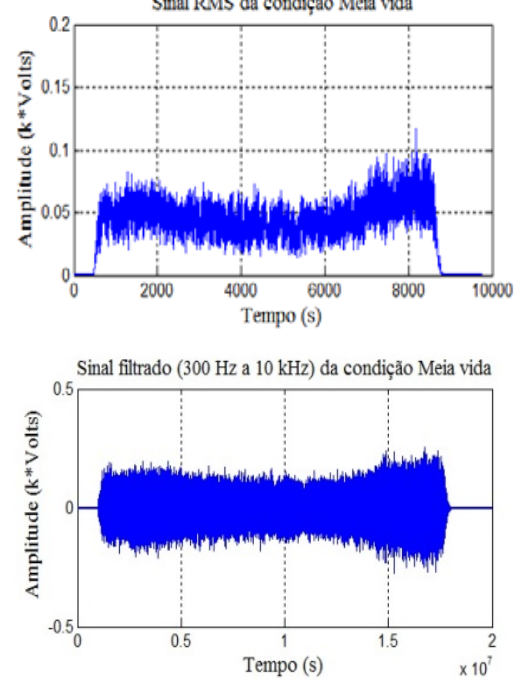
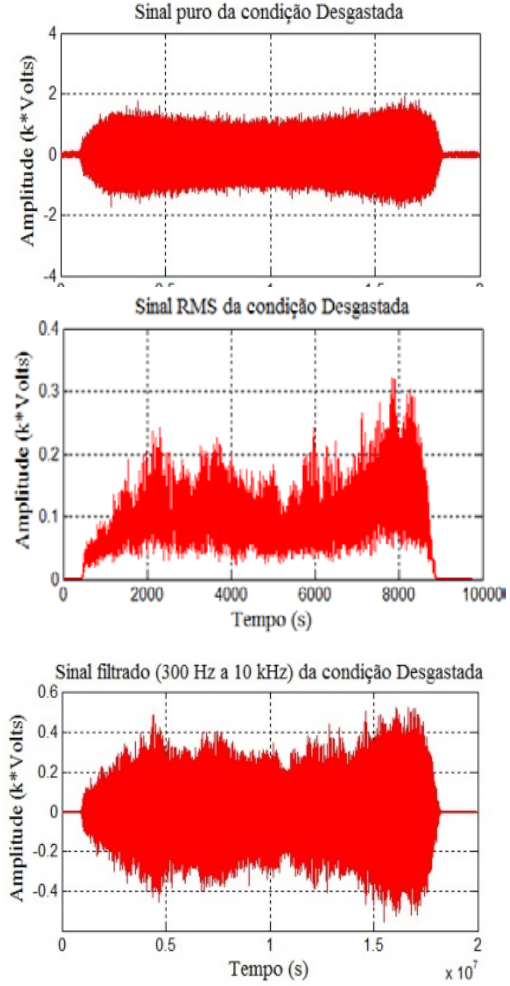

Figura 6: Processamento do sinal de vibração no domínio do tempo

A Figura 7 apresenta o conteúdo em frequência do sinal de vibração inicialmente por meio do espectrograma. O objetivo é avaliar a densidade espectral de energia do sinal, por meio da sua intensidade em cores, sendo possível identificar o comportamento da passada de dressagem. Pode se observar na Figura 7 que a cor vermelha representa a passada válida de dressagem. Nota-se que a intensidade dessa cor aumenta conforme o desgaste ocorre. Dessa forma, na Figura 7 (c) o sinal é mais intenso em termos de cores, ou seja, sua energia está maior. Infere-se que é possível encontrar, também neste último gráfico, faixas de frequência mais altas. 
O estudo do espectro de frequência do sinal de vibração teve como objetivo determinar uma correlação entre características desses sinais com o nível de desgaste do dressador. Observa-se que a atividade vibracional nas frequências mais baixas se intensifica conforme o desgaste avança. Com base nessas observações, foi realizado o estudo em frequência do sinal de vibração por meio da FFT e do PSD utilizando janelas de Hanning em regiões de interesse do sinal, onde foi observada uma correlação mais próxima com o desgaste da ferramenta.
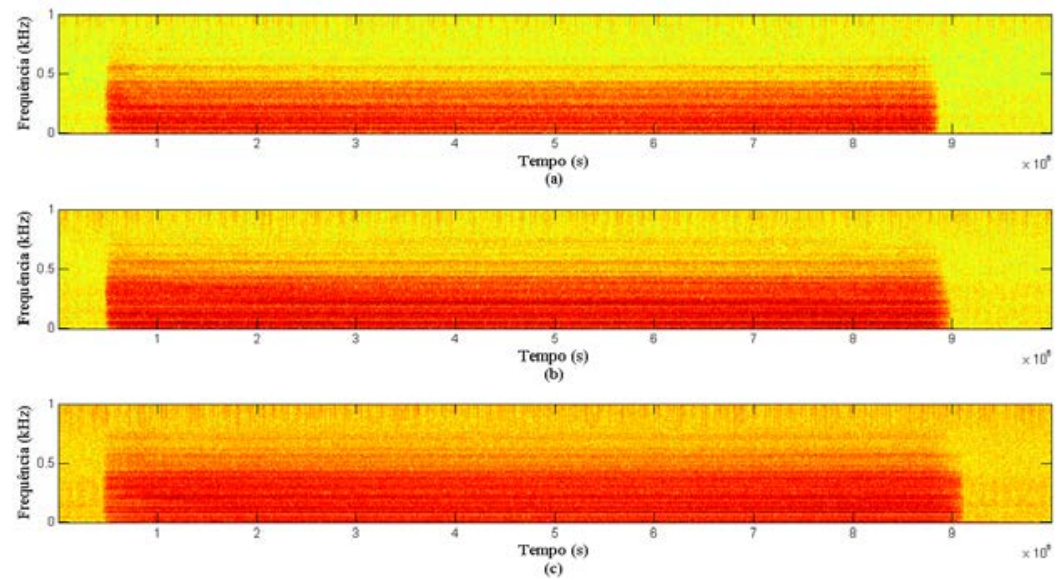

Figura 7: Espectrograma do sinal de vibração. (a) condição nova; (b) condição meia vida e (c) condição desgastada.

Na Figura 8 é apresentado o espectro do sinal vibração com diferentes características ao longo da passada de acordo com a condição da ferramenta. Por exemplo, observam-se baixas amplitudes e sobreposição nos três sinais, nas faixas de frequência de 3 a $5 \mathrm{kHz}$ e de 10 a $15 \mathrm{kHz}$. Por outro lado, nas faixas de 1 a 2 $\mathrm{kHz}$ e de 5 a $10 \mathrm{kHz}$ os sinais apresentam um comportamento interessante para estudo, por suas amplitudes serem maiores e as condições estarem mais bem definidas nessas duas faixas.

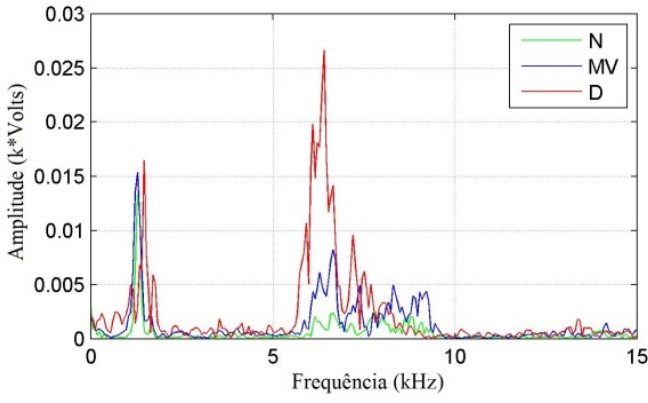

(a)

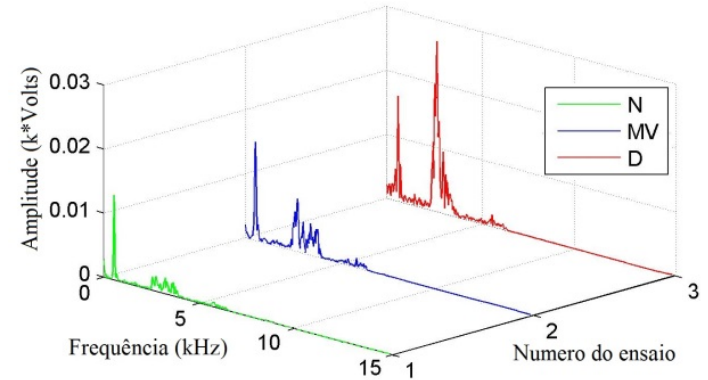

(c)

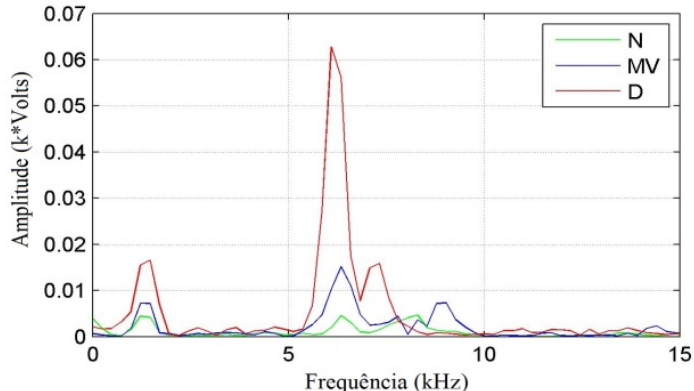

(b)

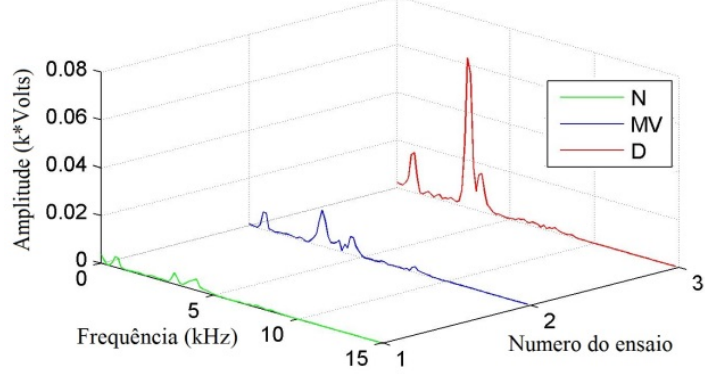

(d)

Figura 8: FFT com janela de $2^{15}$ amostras (a) e (c); FFT com janela de $2^{13}$ amostras (b) e (d), do sinal de aceleração.

O sinal pertencente à condição novo foi o que se manteve com amplitude mais baixa ao longo de toda a passada, apresentando picos mais significativos nas duas faixas de frequência citadas, inferindo a partir disso que houve pouca vibração quando o dressador ainda estava novo. Nos sinais das condições meia-vida e desgastado pode-se observar o aumento da vibração no ensaio. Já que as amplitudes são maiores, o sinal pertencente à condição desgastada, por sua vez, tem um pico bem mais elevado em relação às outras condições, 
caracterizando uma atividade mais intensa do sinal de vibração. Dessa forma infere-se que a vibração aumenta proporcionalmente ao desgaste da ferramenta.

A Figura 9 apresenta o PSD com uma janela de $2^{15}$ e $2^{13}$ amostras do sinal puro de vibração. De maneira geral, observa-se que os três sinais seguem uma tendência ao longo do domínio da frequência e suas amplitudes se intensificam ao longo de toda a passada, conforme ocorre o desgaste da ferramenta. Pode ser observado na Figura 9 (a) e (c) que o espectro se apresenta de forma ruidosa e oscilatória, contendo mais detalhes sobre a passada.

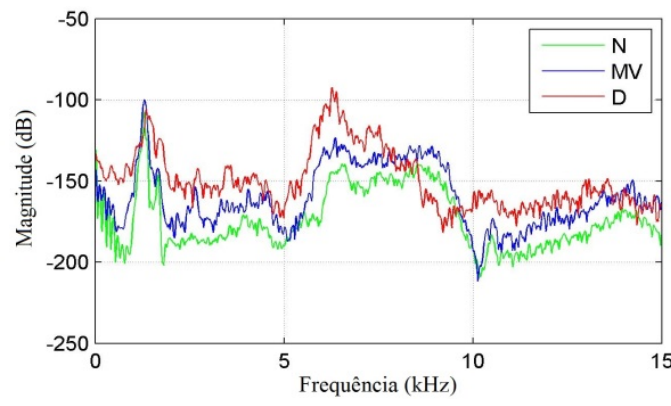

(a)

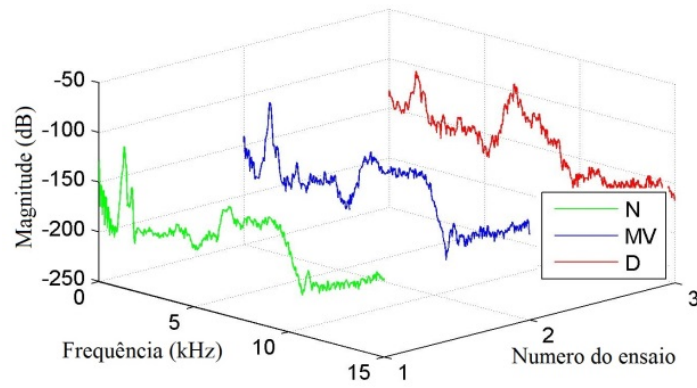

(c)

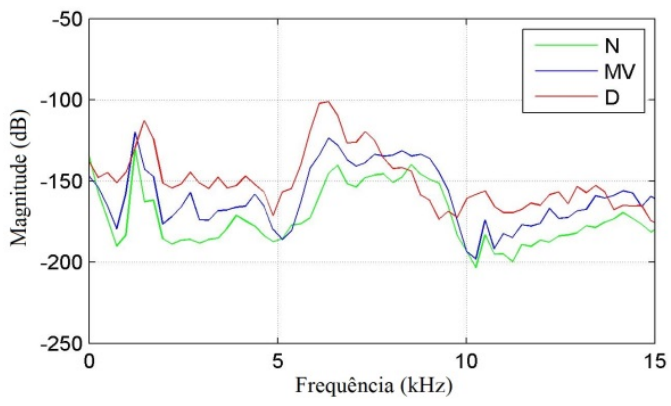

(b)

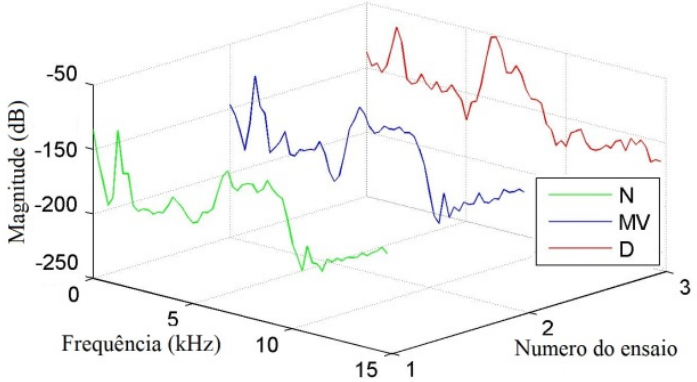

(d)

Figura 9: PSD com janela de $2^{21}$ amostras (a) e (c); e janela de $2^{13}$ amostras (b) e (d), do sinal de aceleração.

Na Figura 9 (b) e (d) os três sinais estão mais bem definidos e classificados de acordo com as condições adotadas, quando comparado com a Figura 9 (a) e (c). Pode-se observar que os sinais apresentam dois picos mais significativos, que se encontram nas faixas de frequência de 1 a $2 \mathrm{kHz}$ e 5 a $10 \mathrm{kHz}$ igualmente ao resultado obtido com a aplicação da FFT (Figura 8). Contudo, na figura 8, podem-se observar outras faixas de frequência que também definem satisfatoriamente as três condições na ordem correta, como na faixa de 3 a $5 \mathrm{kHz}$ por exemplo.

A Figura 10 apresenta, especificamente, o mesmo gráfico da Figura 9 na faixa de frequência entre 1,3 kHz e 8,5 kHz, com objetivo de avaliar com mais detalhes os espectros de vibração.

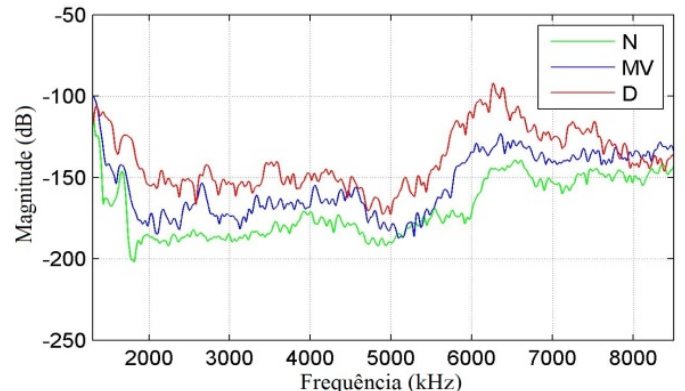

(a)

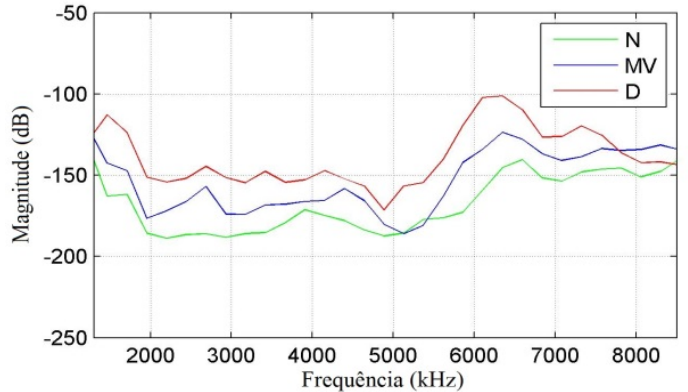

(b)

Figura 10: PSD com janela de $2^{21}$ amostras (a); e janela de $2^{13}$ amostras (b), do sinal de aceleração, entre $1.3 \mathrm{kHz}$ e 8.5 $\mathrm{kHz}$.

É possível notar, a partir dos gráficos (a) e (b) da Figura 10, que o método Welch é sensível à resolução de amostras e, ainda é possível encontrar faixas de frequências mais bem definidas como, por exemplo, de 2 a $4 \mathrm{kHz}$ e de 6 a $7 \mathrm{kHz}$. É possível também observar mais claramente o comportamento dos sinais em cada condição da ferramenta e a sua intensidade em frequência. Pode-se observar que à medida que o dressa- 
dor é utilizado nos ensaios, a vibração aumenta, conforme mostra a amplitude dos sinais.

\section{CONCLUSÕES}

Durante o desenvolvimento deste trabalho foi constatado que ainda existe pouco ou quase nenhum estudo realizado anteriormente, tratando do assunto de monitoramento do desgaste em dressadores por meio da vibração. Sumariamente o processo de retificação é um dos mais importantes da cadeia de processos de manufatura, devido ao seu valor agregado. A operação de dressagem é fundamental para esse processo, e seu monitoramento é de suma importância, visto que erros de dressagem afetam significativamente a qualidade da retificação. O sinal de vibração ainda é pouco utilizado no monitoramento do processo de retificação, dessa forma, é deixada aqui, uma nova contribuição, enfatizando a importância do monitoramento dessa grandeza em particular no processo de dressagem e a sua relação com o desgaste da ferramenta. No caso deste trabalho a inferência foi o desgaste de um dressador de ponta única de diamante CVD.

Dos resultados obtidos, em relação ao desgaste do dressador, pode-se concluir que a área desgastada do diamante CVD comportou-se da forma esperada, apresentando uma característica crescente bem definida, porém devido as diferentes friabilidades dos diamantes, observaram-se variações muito grandes entre as medidas dos ensaios, de acordo com a figura 4.

Observando as Figuras 8, 9 e 10, com objetivo de comparar o espectro de frequência usando FFT com o PSD, usando método Welch, é possível concluir que existe uma correlação entre os dois métodos no que se diz a respeito aos picos de amplitudes. Nota-se que em cada um dos gráficos, os sinais possuem dois picos significativos de amplitudes nas faixas de frequência de 1 a $2 \mathrm{kHz}$ e de 5 a $10 \mathrm{kHz}$. Foi constatado que o sinal pertencente ao nível desgastado tem as maiores amplitudes nos dois gráficos, concluindo que a passada que caracteriza o desgaste do dressador também tem mais atividade de vibração. Nas análises feitas com a FFT (Figura 8) foi possível identificar uma relação proporcional da vibração e desgaste da ferramenta. Contudo, os espectros analisados pelo PSD, usando o método Welch, caracterizam de maneira mais expressiva as três condições da ferramenta, definindo melhor cada uma delas em ordem crescente: novo, meia- vida e desgastado. Isso ocorreu principalmente nas faixas de frequência de 2 a $4 \mathrm{kHz}$ e de 6 a $7 \mathrm{KHz}$, de acordo com a figura 12, o que não foi possível identificar nas análises usando apenas a FFT.

A possibilidade de se monitorar frequências específicas ao longo do domínio da frequência e correlacionar às alterações ocorridas nas faixas de frequências encontradas nesta análise é uma das grandes contribuições desta ferramenta. Esses aspectos representam a diferenciação do presente estudo quando comparado ao trabalho de Martins at al., [18] e [19], pois além de detectar as condições operativas, é possível identificar onde, provavelmente, ocorreu uma falha, com base no padrão de alteração das componentes em frequência do sinal de vibração.

A partir dos resultados obtidos no presente trabalho, um sistema de monitoramento em tempo real pode ser proposto, a fim de permitir uma melhor seleção dos dressadores em função de seu desgaste, contribuindo para a automatização do processo. Uma vez que as faixas de frequências encontradas estão abaixo de $10 \mathrm{kHz}$, isso possibilitará a implementação de hardware de baixo custo para o monitoramento, utilizando filtros digitais nessas faixas previamente selecionadas.

\section{BIBLIOGRAFIA}

[1] WEGENER, K., HOFFMEISTER, H.W., KARPUSCHEWSKI, B. "Conditioning and monitoring of grinding wheels”, CIRP Annals - Manufacturing Technology, v. 60, n. 2, pp. 757-777, Budapest, Maio 2011.

[2] NGUYEN, A. T., BUTLER, D. L. "Correlation of grinding wheel topography and grinding performance: A study from a viewpoint of three-dimensional surface characterization”, Journal of Materials Processing Technology, v. 208, n. 1-3, pp. 14-23, Jan. 2008.

[3] PORZYCKI, J. "Surface-roughness model in traverse grinding”, Archives Civil Mechanics Engineering, v. 5, n. 1, pp. 5-12, 2005.

[4] MARINESCU, I. D., HITCHINER, M., UHLMANN, E., et al. Handbook of machining with grinding wheels, 1 ed, La Palma, CRC Press, 2007.

[5] MALKIN, S., GUO, C. Grinding technology: theory and aplications of machining with abrasives. 2 ed, New York, Industrial Press Inc, 2008.

[6] AGUIAR, P. R., et al., "Monitoring the dressing operation in the grinding process", International Journal of Machining and Machinability of Materials, v. 5, pp. 3-22, 2009.

[7] CALLISTER, W. D. J. Fundamentals of Materials Science and Engineering An Interactive. 4 ed, Nova Jersey, John Wiley \& Sons Inc, 2002. 
[8] MARTINS, C. H. R., AGUIAR, P. R., FRECH, A., et al. "Neural Networks Models for Wear Patterns Recognition of Single-point Dresser”, IFAC Conference on Manufacturing Modelling, Management, v. 46, n. 9, pp. 1524-1529, Mar. 2013.

[9] ANGUS, J. C., HAYMAN, C. C. Low-pressure, metastable growth of diamond and "diamondlike” phases. 1 ed, New York, Science, 1995.

[10] HABRAT, W., BATSCH, A., J., P. "Monitoring of the single-point diamond dresser wear”, Archives of Civil and Mechanical Engineering, v. 1, n.1, pp. 4-15, 2005.

[11] HASSUI, A., DINIZ, A. E., OLIVEIRA, J. F. G., et al. "Experimental evaluation on grinding wheel wear through vibration and acoustic emission”, Wear 217, v. 26. n. 4, pp. 7-14, 1998.

[12] HASSUI, A., DINIZ, A. E. "Correlating surface roughness and vibration on plunge cylindrical grinding of steel”, International Journal of Machine Tools and Manufacture, v. 43, n. 8, pp. 855-862, 2003.

[13] KJÆR, B. “Vibration Measurement and Analysis”, Instrumentation, v. 785, n. 1, pp. 1-40, 1998.

[14] D’ADDONA, D. M, MATARAZZO, D., AGUIAR, P. R., et al., "Neural Networks Tool Condition Monitoring in Single-point Dressing Operations”, Procedia CIRP, v. 41, pp. 431-436, Apr. 2016.

[15] MIRANDA, H. I., ROCHA, C. A., OLIVEIRA, P.C., et al., "Monitoring Single-point Dressers Using Fuzzy Models”, Procedia CIRP, v. 33, pp. 281-286, jun 2015.

[16] ZHANG, C., SHIN, Y. C. "Wear of diamond dresser in laser assisted truing and dressing of vitrified CBN wheels”, International Journal Mach Tools Manufature, v. 43, pp. 41-49., 2003.

[17] MOIA, D. F. G., THOMAZELlA, I. H., AGUIAR, P. R., et al., "Tool condition monitoring of aluminum oxide grinding wheel in dressing operation using acoustic emission and neural networks", Jornal of Brazilian Society of Mechanical Science and Engineering, v. 37, no. 2, pp. 627-640, Apr. 2015.

[18] MARTINS, C. H. R., AGUIAR, P. R., FRECH, A., et al., "Tool Condition Monitoring of Single-Point Dresser Using Acoustic Emission and Neural Networks Models”, IEEE Transactions on Instrumentation and Measurement, v. 63, n. 3, pp. 667-679, Mar. 2014.

[19] MARTINS, C. H. R., AGUIAR, P. R., FRECH, A., et al., "Application of MLP and Kohonen networks for recognition of wear patterns of single-point dressers," IASTED Multiconferences - International Conference Artificial Intelligence and Applications., p. 70 74, 2013.

[20] SOUZA, R. V. C. G., BATISTA, I. H., AGUIAR, P. R., et al., "Study of single point dresser wear by acoustic emission and vibration signal”, In: Procedia XI Brazilian Symposium Intelligence and Automation., v. 11, pp. 1-6, Oct. 2013.

[21] SOUZA, R. V. C. G., ROCHA, C. A., MARCHI, M., AGUIAR, P. R., MARTINS, C. H. R., BIANCHI, E. C, "Tool Condition Monitoring dressing Using Vibration Signals and Neural Networks Models",In: Procedia XX Brazilian Congress of Automatic., v. 20, pp. 1026-1033, Sep. 2014.

[22] LIAO, W. T. “A wavelet-based methodology for grinding wheel condition monitoring”, International Journal of Machine Tools and Manufacture, v. 47, n. 3-4, pp. 580-592, 2007.

[23] DURGUMAHANTI, U. S. P., VIJAYENDER, P. S., VENKATESWARA, R. “A New Model of Grinding Force Prediction and Analysis”, International Journal of Machina Tools \& Manufacture, v. 50, pp. 231-240, 2010

[24] NGUYEN, A. T., BUTLER, D. L. "Correlation of grinding wheel topography and grinding performance: A study from a viewpoint of three-dimensional surface characterisation”, Journal of Materials Processing Technology, v. 208, n. 1-3, pp. 14-23, 2008.

[25] STEMMER, C. E. Ferramentas de Corte II: Brocas, Alargadores, Ferramentas de Roscas, Fresas, Brochas, Rebolos e Abrasivos. 1ed, Florianopolis, Editora da UFSC, 1992.

[26] SALMON, S. C. Modern Grinding Process Technology. 1 ed, Hardcover, 1992.

[27] WEBSTER, J. A. "Directing coolant into the right place, at the right speed, in the right quantity”, CoolGrind Technologies, v. 60, n. 2, pp. 5-17, Feb. 2008.

[28] HEINZEL, C., RICKENS, K. "Engineered wheels for grinding of optical glass”, CIRP Annals Manufacturing Technology, v. 58, n. 1, pp. 315-318, Mar. 2009.

[29] JACKSON, M. J., ROBINSON, G. M.; CHEN, X. "Laser Surface Preparation of Vitrified Grinding Wheels”, Journal of Materials Engineering and Performance, v. 15, pp. 247-250, 2007.

[30] LIN, S. Y., LIU, Y. C., HUANG, C. “An investigation of surface grinding characteristics for titanium alloy with CBN wheel”, Key Engineering Materialss, v. 364-366, pp. 237-242, 2008. 
[31] KOIZUMI, S. "Growth and characterization of phosphorous doped homoepitaxial diamond thin films”, Applied Physics Letters, v. 71, n. 8, pp. 10-65, 1997.

[32] AGUIAR, P. R., SOUZA, A. G. O., BIANCHI, E. C., LEITE, R. R., DOTTO, F. R. L. "Monitoring the dressing operation in the grinding process”, International Journal of Machining and Machinability of Materials, v. 5, n. 1, pp. 3-22, 2009.

[33] XUE, L., NAGHDY, F. "Monitoring of wheel dressing operation for precision grinding”, IEEE International Conference on Industrial Technology ICIT, v. 4, n. 1, pp. 1296-1299, Mar. 2002.

[34] LYONS, R. G. Undestanding Digital Signal Processing, 2 ed, New York, Systems Inc, 2001.

[35] SEMMLOW, J. Biosignal and biomedical image processing MATLAB based applications. 1 ed, Marcel Dekker Inc., 2004.

[36] ALKAN, A., YILMAZ, A. S. "Frequency domain analysis of power system transients using Welch and Yule Walker AR methods”, Energy Conversion and Management, v. 48, n. 1, pp. 2129-2135, 2007.

[37] ZHANG, J., YUAN, Z., HUANG, J., et al. "Quantification of the power changes in BOLD signals using Welch spectrum method during different single-hand motor imageries”, Magnetic Resonance, v. 1, n. 1, pp. 3-14, 2014.

[38] WELCH, P. D. "The use of fast Fourier transform for the estimation of power spectra: a method based on time averaging over short, modified periodograms”, IEEE Transactions Audio Electroacoust, v.1, n.1, pp. 932-945, 1967.

[39] MALKIN, S. Grinding technology: theory and applications of machining with abrasives. 1 ed, Society of Manufacturing Engineers, 1989.

[40] XU, C., LIU, Z., LUO, W. “A frequency band energy analysis of vibration signals for tool condition monitoring”, ICMTMA Inernational Conference Meas Technology Mechatronics Automation, v. 1, pp. 385388, 2009.

[41] INASAKI, I., KARPUSCHEWSKI, B., LEE, H. S. “Grinding chatter - origin and suppression”, Annals of the CIRP - Manufacturing Technology, v. 50, n. 2, pp. 515-534, Nancy, Mai, 2001.

[42] ALTINTAS, Y., WECK, M. "Chatter stability of metal cutting and grinding”, Annals of the CIRPManufacturing Technology, v. 53, n. 2, pp. 619-652, Krakow, Mai. 2004.

[43] DREW, S. J., MANNAN, M. A., ONG, K. L., et al. "The measurement of forces in grinding in the presence of vibration”, International journal of machine tools \& manufacture, v. 41, pp. 509-520, 2001.

[44] TONSHOFF, H. K., FRIEMUTH, T., BECKER, J. C. "Process Monitoring in Grinding”, Annals of the CIRP - Manufacturing Technology, v. 52 . p.551-571, San Sebastian, Apr. 2002.

[45] DAVID, C., WARNECKC, G. "A correlation between cutting process and dynamics of the machinetool-system in grinding”, Transactions Manufacturing Society, v. 24, pp. 51-56, 1996.

[46] LIANG, Z., WANG, X., WU, Y. “An investigation on wear mechanism of resin-bonded diamond wheel in Elliptical Ultrasonic Assisted Grinding (EUAG) of monocrystal sapphire”, Journal of Materials Processing Technology, v. 212, n. 4, pp. 868 - 876, 2012.

[47] XU, C., LIU, Z., LUO, W. “A frequency band energy analysis of vibration signals for tool condition monitoring”, ICMTMA Inernational Conference Meas Technology Mechatronics Automation, v. 1, pp. 385388, 2009.

[48] REDDY, R. Analisys of resonance of a surface grinder, Department of Mechanical Engineering, BKIT,Bhalki, Karnataka, Indian, v.11, pp. 046-053, 2015.

[49] OLIVEIRA, J. F. G., FRANÇA, T.V., WANG, J. P. "Experimental analysis of wheel/workpiece dynamic interactions in grinding”, CIRP Annals - Manufacturing Technology , v.57, pp. 329-332, 2008. 